\title{
Hidden-charm and bottom tetra- and pentaquarks with strangeness in the hadro-quarkonium and compact tetraquark models
}

\author{
J. Ferretti ${ }^{a, b}$ and E. Santopinto ${ }^{c}$ \\ ${ }^{a}$ Center for Theoretical Physics, Sloane Physics Laboratory, Yale University, \\ New Haven, Connecticut 06520-8120, U.S.A. \\ ${ }^{b}$ Department of Physics, University of Jyväskylä, \\ P.O. Box 35 (YFL), 40014 Jyväskylä, Finland \\ ${ }^{c}$ Istituto Nazionale di Fisica Nucleare (INFN), Sezione di Genova, \\ Via Dodecaneso 33, 16146 Genova, Italy \\ E-mail: jacopo.j.ferretti@jyu.fi, elena.santopinto@ge.infn.it
}

ABSTRACT: In two recent papers, we used the hadro-quarkonium model to study the properties of hidden-charm and bottom tetraquarks and pentaquarks. Here, we extend the previous results and calculate the masses of heavy-quarkonium-kaon/hyperon systems. We also compute the spectrum of hidden-charm and bottom tetraquarks with strangeness in the compact tetraquark (diquark-antidiquark) model. If heavy-light exotic systems with non-null strangeness content were to be observed experimentally, it might be possible to distinguish among the large variety of available theoretical pictures for tetra- and pentaquark states and, possibly, rule out those which are not compatible with the data.

KeYwords: Phenomenological Models, QCD Phenomenology

ARXIV EPRINT: 2001.01067 


\section{Contents}

1 Introduction 1

2 Hadro-quarkonium model $\quad 3$

3 Spectra of strange hidden-charm and bottom tetra- and pentaquarks in the hadro-quarkonium model

3.1 Hidden-charm and hidden-bottom pentaquarks with strangeness in the hadro-quarkonium model

3.2 Hidden-charm and hidden-bottom tetraquarks with strangeness in the $\begin{array}{ll}\text { hadro-quarkonium model } & 7\end{array}$

4 Relativized diquark model $\quad 8$

5 Masses of $c s \bar{c} \bar{n}$ and $b s \bar{b} \bar{n}$ states in the compact tetraquark model $\quad 10$

$\begin{array}{lll}5.1 \text { Ground-state energies of } c s \bar{c} \bar{n} \text { and } b s \bar{b} \bar{n} \text { tetraquarks } & 10\end{array}$

$\begin{array}{lll}5.2 & \text { Spectra of } c s \bar{c} \bar{n} \text { and } b s \bar{b} \bar{n} \text { tetraquarks } & 11\end{array}$

6 Conclusion 13

\section{Introduction}

Multiquark states are baryons/mesons which cannot be described in terms of $q q q / q \bar{q}$ degrees of freedom only. They include $X Y Z$ suspected tetraquarks, like the $X(3872)$ [now $\chi_{\mathrm{c} 1}(3872)$ ] [1-3] and $X(4274)$ [also known as $\chi_{\mathrm{c} 1}(4274)$ ] [4-6], and pentaquark states. The latter were recently discovered by $\mathrm{LHCb}$ in $\Lambda_{\mathrm{b}} \rightarrow J / \psi \Lambda^{*}$ and $\Lambda_{\mathrm{b}} \rightarrow P_{\mathrm{c}}^{+} K^{-} \rightarrow(J / \psi p) K^{-}$ decays $[7,8]$. The structure of $X Y Z$ tetraquarks and $P_{\mathrm{c}}$ pentaquarks is still unclear. This is why there are several alternative models to explain their properties. For a review, see refs. [9-12]. To distinguish among the different pictures (molecular model, diquark model, unquenched quark model, ...) one should compare their theoretical predictions for the spectrum, decay amplitudes, production cross-sections, and so on, with the experimental data.

A clean way to discriminate among the previous theoretical interpretations for suspected $X Y Z$ tetraquarks was suggested in ref. [13]. There, Voloshin pointed out that if $Z_{\mathrm{c}}$ resonances exist then, because of the $\mathrm{SU}(3)_{\mathrm{f}}$ symmetry, one may also expect the emergence of their strange partners, $Z_{\mathrm{cs}}$ [13]. The author also argued that the one-pionexchange interaction of the meson-meson molecular model is impossible between strange and nonstrange heavy mesons, like $B$ and $B_{\mathrm{s}}$ [13]. Hidden-charm and bottom mesons with strangeness are also forbidden in the context of the Unquenched Quark Model (UQM) formalism. Indeed, one cannot dress heavy quarkonium $Q \bar{Q}$ states with $Q \bar{s}-n \bar{Q}$ or $Q \bar{n}-s \bar{Q}$ higher Fock components (where $n=u$ or $d$ ) by creating a light $n \bar{n}$ or $s \bar{s}$ pair with vacuum quantum numbers. Therefore, hidden-charm and bottom tetraquark states with non-null 

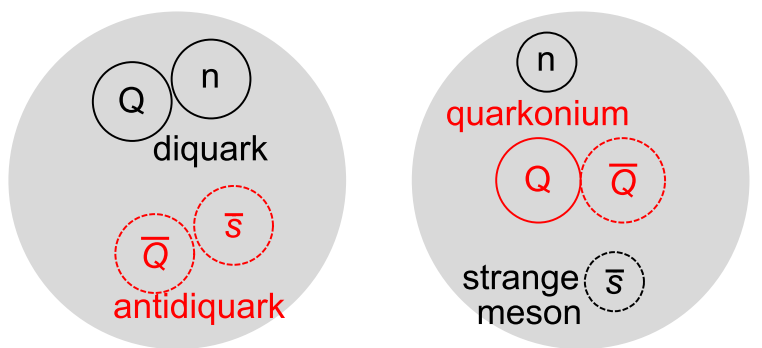

Figure 1. Schematic representation of heavy-light hadro-quarkonium (right) and compact tetraquark (left) states.

strangeness content cannot take place neither in the UQM [14-27] nor in the molecular model [28-34] interpretations. On the contrary, these exotic configurations are expected (if above threshold) both in the compact tetraquark [35-50] and hadro-quarkonium [13, 51-66] models. See figure 1. In light of this, the experimental observation of $X Y Z$ states with non-null strangeness content would make it possible to rule out a few possible theoretical interpretations for tetraquarks. Voloshin did not compute the spectrum of $Z_{\mathrm{cs}}$ states, but only discussed phenomenological indications for the emergence of those states [13]. The study of their spectrum and that of their pentaquark counterparts is thus the subject of the present manuscript.

Here, we extend the hadro-quarkonium model findings of refs. [64, 65] and calculate the spectrum of hidden-charm and bottom tetraquarks and pentaquarks with strangeness. The hadro-quarkonium picture was developed to explain the experimental observation of heavy-light tetraquark candidates characterized by peculiar properties [53, 67]. Firstly, these exotics are supposed not to be particularly close to a specific heavy-light mesonmeson threshold, unlike $D^{0} \bar{D}^{* 0}$ in the $X(3872)$ case. Secondly, such states may decay into heavy quarkonia plus one or more light mesons, like $\eta_{\mathrm{c}}+\eta$. Even though it was meant for the description of tetraquarks, the hadro-quarkonium model can be easily extended to the baryon sector to study pentaquarks [59-61, 65].

We also compute the masses of heavy-light tetraquarks with non-null strangeness content in the compact tetraquark model of refs. [46, 48, 50]. In the compact tetraquark model, heavy-light $q Q \bar{q} \bar{Q}$ states are modeled as the bound states of a diquark, $q Q$, antidiquark, $\bar{q} \bar{Q}$, pair. The diquark constituents are treated as inert against internal spatial excitations. Their binding is the consequence of one-gluon-exchange forces and their relative dynamics can be described in terms of a relative coordinate $\mathbf{r}_{\text {rel }}$. The calculation of the spectrum of compact pentaquark configurations in the diquark model is more difficult than that of compact tetraquarks because one has to deal with a three-body problem instead of a two-body one; moreover, one also has to consider both diquark-diquark and diquark-antiquark interactions. This is why here we do not provide results for compact (diquark-diquark-antiquark) pentaquarks, which will be the subject of a subsequent paper.

Our predictions for strange hidden-charm and bottom tetraquarks and, especially, those for $P_{\mathrm{c}}$ and $P_{\mathrm{b}}$ pentaquarks with non-null strangeness content may soon be tested by $\mathrm{LHCb}$. 


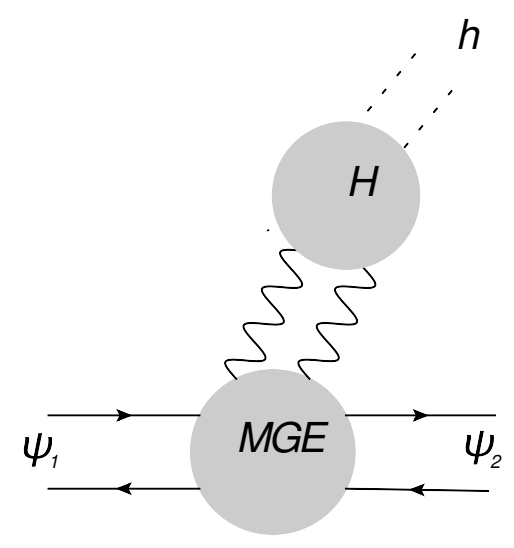

Figure 2. Hidden-flavor transition $\psi_{1} \rightarrow \psi_{2}+h$ in the QCD multipole expansion. Here, $\psi_{1}$ and $\psi_{2}$ are the initial and final charmonium states, $h$ light hadron(s). The two vertices are those of the multipole gluon emission, $M G E$, and hadronization, $H$. Picture from ref. [64]; Elsevier Copyright.

\section{Hadro-quarkonium model}

The possible existence of binding mechanisms of charmonium states in light-quark matter was discussed long ago [68-70] in terms of the interaction of charmonium inside nuclei. The idea of hadro-charmonium (hadro-quarkonium) bound states resembles the previous one.

Hadro-quarkonia are heavy-light tetra- or pentaquark configurations, where a compact $Q \bar{Q}$ state (with $Q=c$ or $b$ ), labelled as $\psi$ in the following, is embedded in light hadronic matter, $\mathcal{H}=q q q$ or $q \bar{q}$ (where $q=u, d$ or $s$ ) [13,51-66]. The heavy and light constituents, $\psi$ and $\mathcal{H}$, develop an attractive force, which is the result of multiple-gluon exchange between them. Such interaction, $H_{\text {eff }}$, can be written in terms of the multipole expansion in QCD [71-73]. In particular, if one considers as leading term the $E 1$ interaction with chromo-electric fields $\mathbf{E}$ and $\mathbf{E}^{\prime}$ [53, 69], one gets the effective Hamiltonian

$$
H_{\mathrm{eff}}=-\frac{1}{2} \alpha_{\psi \psi^{\prime}} \mathbf{E} \cdot \mathbf{E}^{\prime}
$$

where $\alpha_{\psi \psi^{\prime}}$ is the so-called heavy quarkonium chromo-electric polarizability. By making use of additional approximations, $H_{\text {eff }}$ can be further reduced to a simple square-well potential [53, 64, 65],

$$
V_{\mathrm{hq}}(r)=\left\{\begin{array}{cl}
-\frac{2 \pi \alpha_{\psi \psi} M_{\mathcal{H}}}{3 R_{\mathcal{H}}^{3}} & \text { for } r<R_{\mathcal{H}} \\
0 & \text { for } r>R_{\mathcal{H}}
\end{array},\right.
$$

where $R_{\mathcal{H}}=R_{\mathcal{B}}$ or $R_{\mathcal{M}}$ is the light baryon/meson radius. Eq. (2.2) can be plugged into a Schrödinger equation and solved for light hadron-heavy quarkonium systems.

There are four quantities to be given as input in the calculation. They are the masses $M_{\psi}$ and $M_{\mathcal{H}}$, the radius $R_{\mathcal{H}}$, and the diagonal chromo-electric polarizability, $\alpha_{\psi \psi}$. See table 1. The values of $M_{\psi}$ and $M_{\mathcal{H}}$ are extracted from the PDG [74].

In principle, non-diagonal quarkonium chromo-electric polarizabilities, $\alpha_{\psi \psi^{\prime}}$, can be fitted to the data by considering $\psi \rightarrow \psi^{\prime}+h$ hadronic transitions [67, 75]; see figure 2 . However, no experimental information can be used to estimate the $\alpha_{\psi \psi}$ 's. Therefore, the 


\begin{tabular}{|cccc|}
\hline Parameter & Value & Parameter & Value \\
\hline$\alpha_{\psi \psi}(1 P)_{c \bar{c}}$ & $11 \mathrm{GeV}^{-3}$ & $\alpha_{\psi \psi}(2 S)_{c \bar{c}}$ & $18 \mathrm{GeV}^{-3}$ \\
$\alpha_{\psi \psi}^{(1)}(1 P)_{b \bar{b}}$ & $14 \mathrm{GeV}^{-3}$ & $\alpha_{\psi \psi}^{(1)}(2 S)_{b \bar{b}}$ & $23 \mathrm{GeV}^{-3}$ \\
$\alpha_{\psi \psi}^{(2)}(1 P)_{b \bar{b}}$ & $21 \mathrm{GeV}^{-3}$ & $\alpha_{\psi \psi}^{(2)}(2 S)_{b \bar{b}}$ & $33 \mathrm{GeV}^{-3}$ \\
$R_{\Sigma}$ & $0.863 \mathrm{fm}$ & $R_{\Xi}$ & $0.841 \mathrm{fm}$ \\
$R_{K}$ & $0.560 \mathrm{fm}$ & $R_{K^{*}}$ & $0.729 \mathrm{fm}$ \\
\hline
\end{tabular}

Table 1. Hadro-quarkonium model. Input values and parameters.

diagonal chromo-electric polarizabilities, $\alpha_{\psi \psi}(n \ell)$, where $n$ and $\ell$ are the radial quantum number and orbital angular momentum of $\psi$, respectively, have to be extracted from the phenomenology. In the case of charmonia, we consider [65]: $\alpha_{\psi \psi}(1 P)_{c \bar{c}}=11 \mathrm{GeV}^{-3}$ and $\alpha_{\psi \psi}(2 S)_{c \bar{c}}=18 \mathrm{GeV}^{-3}$. In the case of bottomonia, we make use of two sets of values for the chromo-electric polarizabilities. They are [65]: $\alpha_{\psi \psi}^{(1)}(1 P)_{b \bar{b}}=14 \mathrm{GeV}^{-3}$ and $\alpha_{\psi \psi}^{(1)}(2 S)_{b \bar{b}}=$ $23 \mathrm{GeV}^{-3} ; \alpha_{\psi \psi}^{(2)}(1 P)_{b \bar{b}}=21 \mathrm{GeV}^{-3}$ and $\alpha_{\psi \psi}^{(2)}(2 S)_{b \bar{b}}=33 \mathrm{GeV}^{-3}$. We also need the strange mesons' and hyperons' radii. While for the kaon we can use the well-established value of the $K^{ \pm}$charge radius reported on the PDG [74], $R_{K}=0.560 \pm 0.031 \mathrm{fm}$, in the $\Sigma, \Xi$ and $K^{*}$ cases the situation is different. ${ }^{1}$ Indeed, due to the lack of well-established experimental data, we are forced to extract $R_{\Sigma}, R_{\Xi}$ and $R_{K^{*}}$ from phenomenological estimates. For example, see refs. [76-80]. Following ref. [79], we have: $R_{\Sigma}=\frac{1}{2}\left(R_{\Sigma^{+}}+R_{\Sigma^{-}}\right)=0.863 \mathrm{fm}$; $R_{\Xi}=0.841 \mathrm{fm}$. The $K^{*}(892)$ 's radius is calculated in the relativized quark model for mesons of ref. [76]: $R_{K^{*}}=0.729 \mathrm{fm}$.

Finally, the hadro-quarkonium quantum numbers are obtained by combining those of the hadrons $\psi$ and $\mathcal{H}$,

$$
\left|\Phi_{\mathrm{hq}}\right\rangle=\left|\left(L_{\psi}, S_{\psi}\right) J_{\psi} ;\left(L_{\mathcal{H}}, S_{\mathcal{H}}\right) J_{\mathcal{H}} ;\left(J_{\mathrm{hq}}, \ell_{\mathrm{hq}}\right) J_{\text {tot }}^{P}\right\rangle .
$$

Here, $\mathbf{J}_{\mathrm{hq}}=\mathbf{J}_{\psi}+\mathbf{J}_{\mathcal{H}}$, the hadro-quarkonium parity is $P=(-1)^{\ell_{\mathrm{hq}}} P_{\psi} P_{\mathcal{H}}$, and $\ell_{\mathrm{hq}}$ is the relative angular momentum between $\psi$ and $\mathcal{H}$. From now on, unless explicitly indicated, we assume that $\ell_{\mathrm{hq}}=0$.

\section{Spectra of strange hidden-charm and bottom tetra- and pentaquarks in the hadro-quarkonium model}

In this section, we discuss our results for the spectrum of heavy quarkonium-strange hadron bound states.

The binding energies are computed in the hadro-quarkonium model of section 2 and refs. [53, 64, 65] by solving the two-body eigenvalue problem of eq. (2.2) via a finite differences algorithm [81, Vol. 3, section 16-6]. As a check, the same results are also obtained

\footnotetext{
${ }^{1}$ The values of the proton and kaon radii reported by the PDG [74] can be regarded as reliable, because they are the result of the average over several measurements. On the contrary, the value of the $\Sigma^{-}$radius from the PDG is the outcome of a single experiment; moreover, there is no available data for the charge radius of the $\Sigma^{+}$. This is why here we do not extract $R_{\Sigma}$ from the PDG.
} 


\begin{tabular}{|ccccc|}
\hline Composition & Quark content & $\alpha_{\psi \psi}(n \ell)\left[\mathrm{GeV}^{-3}\right]$ & $J_{\text {tot }}^{P}$ & Mass (Binding) $[\mathrm{MeV}]$ \\
\hline$\chi_{\mathrm{c} 0}(1 P) \otimes \Sigma$ & $n n s c \bar{c}$ & 11 & $\frac{1}{2}^{+}$ & $4440(-166)$ \\
$\eta_{\mathrm{c}}(2 S) \otimes \Sigma$ & $n n s c \bar{c}$ & 18 & $\frac{1}{2}^{-}$ & $4474(-355)$ \\
$\psi(2 S) \otimes \Sigma$ & $n n s c \bar{c}$ & 18 & $\frac{1}{2}^{-}$or $\frac{3}{2}^{-}$ & $4522(-355)$ \\
$\chi_{\mathrm{c} 1}(1 P) \otimes \Sigma$ & $n n s c \bar{c}$ & 11 & $\frac{1}{2}^{+}$or $\frac{3}{2}^{+}$ & $4535(-166)$ \\
$h_{\mathrm{c}}(1 P) \otimes \Sigma$ & $n n s c \bar{c}$ & 11 & $\frac{1}{2}^{+}$or $\frac{3}{2}^{+}$ & $4550(-167)$ \\
$\chi_{\mathrm{c} 2}(1 P) \otimes \Sigma$ & $n n s c \bar{c}$ & 11 & $\frac{3}{2}^{+}$or $\frac{5}{2}^{+}$ & $4580(-167)$ \\
{$\left[\eta_{\mathrm{c}}(2 S) \otimes \Sigma\right]_{\ell_{\mathrm{hq}}=1}$} & $n n s c \bar{c}$ & 18 & $\frac{1}{2}^{+}$or $\frac{3}{2}^{+}$ & $4653(-175)$ \\
{$[\psi(2 S) \otimes \Sigma]_{\ell_{\mathrm{hq}}=1}$} & $n n s c \bar{c}$ & 18 & $\frac{1}{2}^{+}, \frac{3}{2}^{+}$or $\frac{5}{2}^{+}$ & $4701(-176)$ \\
\hline$\eta_{\mathrm{c}}(2 S) \otimes \Xi$ & $n s s c \bar{c}$ & 18 & $\frac{1}{2}^{-}$ & $4500(-459) ; 4955(-5)$ \\
$\chi_{\mathrm{c} 0}(1 P) \otimes \Xi$ & $n s s c \bar{c}$ & 11 & $\frac{1}{2}^{+}$ & $4510(-226)$ \\
$\psi(2 S) \otimes \Xi$ & $n s s c \bar{c}$ & 18 & $\frac{1}{2}^{-}$or $\frac{3}{2}^{-}$ & $4548(-460) ; 5002(-5)$ \\
$\chi_{\mathrm{c} 1}(1 P) \otimes \Xi$ & $n s s c \bar{c}$ & 11 & $\frac{1}{2}^{+}$or $\frac{3}{2}^{+}$ & $4605(-227)$ \\
$h_{\mathrm{c}}(1 P) \otimes \Xi$ & $n s s c \bar{c}$ & 11 & $\frac{1}{2}^{+}$or $\frac{3}{2}^{+}$ & $4620(-227)$ \\
$\chi_{\mathrm{c} 2}(1 P) \otimes \Xi$ & $n s s c \bar{c}$ & 11 & $\frac{3}{2}^{+}$or $\frac{5}{2}^{+}$ & $4650(-228)$ \\
{$\left[\eta_{\mathrm{c}}(2 S) \otimes \Xi\right]_{\ell_{\mathrm{hq}}=1}$} & $n s s c \bar{c}$ & 18 & $\frac{1}{2}^{+}$or $\frac{3}{2}^{+}$ & $4685(-274)$ \\
{$[\psi(2 S) \otimes \Xi]_{\ell_{\mathrm{hq}}=1}$} & $n s s c \bar{c}$ & 18 & $\frac{1}{2}^{+}, \frac{3}{2}^{+}$or $\frac{5}{2}^{+}$ & $4733(-275)$ \\
\hline
\end{tabular}

Table 2. Hadro-quarkonium model predictions for charmonium- $\Sigma$ and $\Xi$ bound states. The pentaquark binding energies and masses (5th column) are calculated with the values of the chromoelectric polarizabilities $\alpha_{\psi \psi}(n \ell)$ (3rd column). Here, $n=u$ or $d$. The bound states are $S$-wave configurations (i.e. $\ell_{\mathrm{hq}}=0$ ), except where explicitly indicated. In some cases, the $V_{\text {hq }}$ potential well is deep enough to give rise to a heavy-quarkonium-baryon bound state and its radial excitation. In this instance, the masses of both the ground-state and excited hadro-quarkonium configurations are reported in the fifth column.

by means of a numerical code based on the Multhopp method; see [82, section 2.4]. The values of the heavy quarkonium chromo-electric polarizabilities and light hadron radii used here are given in table 1.

\subsection{Hidden-charm and hidden-bottom pentaquarks with strangeness in the hadro-quarkonium model}

The first step of our investigation is the study of heavy quarkonium-hyperon bound states. Our findings are enlisted in tables 2 and 3.

It is worth noting that: I) According to our predictions, heavy-quarkonium-hyperon states may be deeply bound; II) In some cases, the $V_{\text {hq }}$ potential well is deep enough to give rise to a heavy-quarkonium-baryon bound state and its radial excitation; III) Our results show a strong dependence on the hyperon's radius, $R_{\mathcal{B}}$. See eq. (2.2). The theoretical predictions for $R_{\mathcal{B}}$ 's are highly model dependent and span a relatively wide range [77-80]. 


\begin{tabular}{|c|c|c|c|c|}
\hline Composition & Quark content & $\alpha_{\psi \psi}(n \ell)\left[\mathrm{GeV}^{-3}\right]$ & $J_{\text {tot }}^{P}$ & Mass (Binding) $[\mathrm{MeV}]$ \\
\hline$\eta_{\mathrm{b}}(2 S) \otimes \Sigma$ & $n n s b \bar{b}$ & 23 & $\frac{1}{2}^{-}$ & $10671(-519) ; 11118(-72)$ \\
\hline$\Upsilon(2 S) \otimes \Sigma$ & $n n s b \bar{b}$ & 23 & $\frac{1}{2}^{-}$or $\frac{3}{2}^{-}$ & $10695(-519) ; 11142(-72)$ \\
\hline$\chi_{\mathrm{b} 0}(1 P) \otimes \Sigma$ & $n n s b \bar{b}$ & 14 & $\frac{1}{2}^{+}$ & $10784(-267)$ \\
\hline$\chi_{\mathrm{b} 1}(1 P) \otimes \Sigma$ & $n n s b \bar{b}$ & 14 & $\frac{1}{2}^{+}$or $\frac{3}{2}^{+}$ & $10817(-267)$ \\
\hline$h_{\mathrm{b}}(1 P) \otimes \Sigma$ & $n n s b \bar{b}$ & 14 & $\frac{1}{2}^{+}$or $\frac{3}{2}^{+}$ & $10824(-267)$ \\
\hline$\chi_{\mathrm{b} 2}(1 P) \otimes \Sigma$ & $n n s b \bar{b}$ & 14 & $\frac{3}{2}^{+}$or $\frac{5}{2}^{+}$ & $10836(-267)$ \\
\hline$\left[\eta_{\mathrm{b}}(2 S) \otimes \Sigma\right]_{\ell_{\mathrm{hq}}=1}$ & $n n s b \bar{b}$ & 23 & $\frac{1}{2}^{+}$or $\frac{3}{2}^{+}$ & $10840(-350)$ \\
\hline$[\Upsilon(2 S) \otimes \Sigma]_{\ell_{\mathrm{hq}}=1}$ & $n n s b \bar{b}$ & 23 & $\frac{1}{2}^{+}, \frac{3}{2}^{+}$or $\frac{5}{2}^{+}$ & $10864(-350)$ \\
\hline$\eta_{\mathrm{b}}(2 S) \otimes \Sigma$ & $n n s b \bar{b}$ & 33 & $\frac{1}{2}^{-}$ & $10383(-807) ; 10885(-305)$ \\
\hline$\Upsilon(2 S) \otimes \Sigma$ & $n n s b \bar{b}$ & 33 & $\frac{1}{2}^{-}$or $\frac{3}{2}^{-}$ & $10407(-808) ; 10909(-306)$ \\
\hline$\left[\eta_{\mathrm{b}}(2 S) \otimes \Sigma\right]_{\ell_{\mathrm{hq}}=1}$ & $n n s b \bar{b}$ & 33 & $\frac{1}{2}^{+}$or $\frac{3}{2}^{+}$ & $10564(-626) ; 11175(-15)$ \\
\hline$[\Upsilon(2 S) \otimes \Sigma]_{\ell_{\mathrm{hq}}=1}$ & $n n s b \bar{b}$ & 33 & $\frac{1}{2}^{+}, \frac{3}{2}^{+}$or $\frac{5}{2}^{+}$ & $10588(-626) ; 11199(-15)$ \\
\hline$\chi_{\mathrm{b} 0}(1 P) \otimes \Sigma$ & $n n s b \bar{b}$ & 21 & $\frac{1}{2}^{+}$ & $10588(-462) ; 11016(-34)$ \\
\hline$\chi_{\mathrm{b} 1}(1 P) \otimes \Sigma$ & $n n s b \bar{b}$ & 21 & $\frac{1}{2}^{+}$or $\frac{3}{2}^{+}$ & $10622(-462) ; 11049(-34)$ \\
\hline$h_{\mathrm{b}}(1 P) \otimes \Sigma$ & $n n s b \bar{b}$ & 21 & $\frac{1}{2}^{+}$or $\frac{3}{2}^{+}$ & $10628(-462) ; 11056(-34)$ \\
\hline$\chi_{\mathrm{b} 2}(1 P) \otimes \Sigma$ & $n n s b \bar{b}$ & 21 & $\frac{3}{2}^{+}$or $\frac{5}{2}^{+}$ & $10641(-462) ; 11069(-34)$ \\
\hline$\eta_{\mathrm{b}}(2 S) \otimes \Xi$ & $n s s b \bar{b}$ & 23 & $\frac{1}{2}^{-}$ & $10664(-657) ; 11126(-194)$ \\
\hline$\Upsilon(2 S) \otimes \Xi$ & $n s s b \bar{b}$ & 23 & $\frac{1}{2}^{-}$or $\frac{3}{2}^{-}$ & $10688(-657) ; 11150(-195)$ \\
\hline$\chi_{\mathrm{b} 0}(1 P) \otimes \Xi$ & $n s s b \bar{b}$ & 14 & $\frac{1}{2}^{+}$ & $10832(-349)$ \\
\hline$\left[\eta_{\mathrm{b}}(2 S) \otimes \Xi\right]_{\ell_{\mathrm{hq}}=1}$ & $n s s b \bar{b}$ & 23 & $\frac{1}{2}^{+}$or $\frac{3}{2}^{+}$ & $10833(-488)$ \\
\hline$[\Upsilon(2 S) \otimes \Xi]_{\ell_{\mathrm{hq}}=1}$ & $n s s b \bar{b}$ & 23 & $\frac{1}{2}^{+}, \frac{3}{2}^{+}$or $\frac{5}{2}^{+}$ & $10857(-488)$ \\
\hline$\chi_{\mathrm{b} 1}(1 P) \otimes \Xi$ & $n s s b \bar{b}$ & 14 & $\frac{1}{2}^{+}$or $\frac{3}{2}^{+}$ & $10865(-349)$ \\
\hline$h_{\mathrm{b}}(1 P) \otimes \Xi$ & $n s s b \bar{b}$ & 14 & $\frac{1}{2}^{+}$or $\frac{3}{2}^{+}$ & $10872(-349)$ \\
\hline$\chi_{\mathrm{b} 2}(1 P) \otimes \Xi$ & $n s s b \bar{b}$ & 14 & $\frac{3}{2}^{+}$or $\frac{5}{2}^{+}$ & $10885(-349)$ \\
\hline$\eta_{\mathrm{b}}(2 S) \otimes \Xi$ & $n s s b \bar{b}$ & 33 & $\frac{1}{2}^{-}$ & $10315(-1006) ; 10818(-502)$ \\
\hline$\Upsilon(2 S) \otimes \Xi$ & $n s s b \bar{b}$ & 33 & $\frac{1}{2}^{-}$or $\frac{3}{2}^{-}$ & $10339(-1006) ; 10842(-503)$ \\
\hline$\left[\eta_{\mathrm{b}}(2 S) \otimes \Xi\right]_{\ell_{\mathrm{hq}}=1}$ & $n s s b \bar{b}$ & 33 & $\frac{1}{2}^{+}$or $\frac{3}{2}^{+}$ & $10495(-826) ; 11137(-183)$ \\
\hline$[\Upsilon(2 S) \otimes \Xi]_{\ell_{\mathrm{hq}}=1}$ & $n s s b \bar{b}$ & 33 & $\frac{1}{2}^{+}, \frac{3}{2}^{+}$or $\frac{5}{2}^{+}$ & $10519(-826) ; 11161(-184)$ \\
\hline$\chi_{\mathrm{b} 0}(1 P) \otimes \Xi$ & $n s s b \bar{b}$ & 21 & $\frac{1}{2}^{+}$ & $10593(-588) ; 11044(-138)$ \\
\hline$\chi_{\mathrm{b} 1}(1 P) \otimes \Xi$ & $n s s b \bar{b}$ & 21 & $\frac{1}{2}^{+}$or $\frac{3}{2}^{+}$ & $10627(-588) ; 11077(-138)$ \\
\hline$h_{\mathrm{b}}(1 P) \otimes \Xi$ & $n s s b \bar{b}$ & 21 & $\frac{1}{2}^{+}$or $\frac{3}{2}^{+}$ & $10633(-588) ; 11083(-138)$ \\
\hline$\chi_{\mathrm{b} 2}(1 P) \otimes \Xi$ & $n s s b \bar{b}$ & 21 & $\frac{3}{2}^{+}$or $\frac{5}{2}^{+}$ & $10646(-588) ; 11096(-138)$ \\
\hline
\end{tabular}

Table 3. As table 2, but for bottomonium- $\Sigma$ and $\Xi$ bound states. 
However, the use of different values of the hyperon's radius does not change our first conclusion qualitatively. As an example, we consider the $\eta_{\mathrm{c}}(2 S) \otimes \Sigma$ state. If we extract the value of the $\Sigma$ radius from ref. [80], $R_{\Sigma}=\frac{1}{2}\left(R_{\Sigma^{+}}+R_{\Sigma^{-}}\right)=0.91 \mathrm{fm}$, we get a binding energy $B_{\eta_{\mathrm{c}}(2 S) \otimes \Sigma}=-294 \mathrm{MeV}$; if we use the experimental value [74], $R_{\Sigma}=R_{\Sigma^{-}}=0.780 \mathrm{fm}$, we obtain $B_{\eta_{\mathrm{c}}(2 S) \otimes \Sigma}=-492 \mathrm{MeV}$. The previous results can be compared to our prediction from table $2, B_{\eta_{c}(2 S) \otimes \Sigma}=-355 \mathrm{MeV}$, calculated with $R_{\Sigma}=\frac{1}{2}\left(R_{\Sigma^{+}}+R_{\Sigma^{-}}\right)=0.863 \mathrm{fm}$ [79]; IV) In bottomonium-hyperon configurations, the presence of a heavier (nonrelativistic) $b \bar{b}$ pair is expected to make the hadro-bottomonium system more stable than the hadrocharmonium one due to kinetic energy suppression. This is why the strange hidden-bottom pentaquarks are more tightly bound than their hidden-charm counterparts; V) If we consider the second set of values for the bottomonium chromo-electric polarizabilities of table 1 , we get bottomonium- $\Sigma$ bound states characterized by very large binding energies. The hadro-quarkonium picture may break down in these specific cases. Thus, one may have to consider the possibility of a mixing between hadro-quarkonium and compact five-quark components:

$$
H=\left(\begin{array}{cc}
H_{\mathrm{hq}} & V_{\text {mixing }} \\
V_{\text {mixing }} & H_{\text {compact }}
\end{array}\right) .
$$

Here, $H_{\mathrm{hq}}=V_{\mathrm{hq}}+T_{\mathrm{hq}}$ is the hadro-quarkonium Hamiltonian, with $T_{\mathrm{hq}}$ being the $\psi \mathcal{H}$ relative kinetic energy and $V_{\text {hq }}$ the potential of eq. (2.2); $H_{\text {compact }}$ is an effective Hamiltonian, which describes a compact five-quark system; $V_{\text {mixing }}$ is an off-diagonal interaction, which mixes hadro-quarkonium and compact five-quark components.

\subsection{Hidden-charm and hidden-bottom tetraquarks with strangeness in the hadro-quarkonium model}

As a second step, we study heavy quarkonium-kaon and $K^{*}$ configurations. Our findings are enlisted in tables 4 and 5 .

Heavy quarkonium-kaon bound states show similar features as the heavy-light pentaquarks of section 3.1. In particular, one can notice that: I) The hadro-quarkonium interaction, eq. (2.2), may determine the emergence of deeply-bound charmonium-kaon tetraquark configurations; II) Even more stable configurations are the bottomonium-kaon ones; III) In both previous cases, if one substitutes the kaon with the $K^{*}$, one obtains extremely stable systems. As discussed in section 3.1, a more realistic description of $\psi K^{*}$ systems may be accomplished by making use of the Hamiltonian (3.1), where one also takes mixing effects between hadro-quarkonium and compact tetraquark components into account. Compact heavy-light tetraquarks have been extensively studied. For example, see the potential model calculations of refs. [41, 42, 45, 48-50] and sections 4 and 5.

The quality of the approximation of neglecting mixing effects between the heavy, $\psi$, and the light, $\mathcal{H}$, hadron components in the hadro-charmonium states of table 4 can be evaluated by calculating the wave function overlap of the previous components at the hadro-quarkonium center

$$
P_{\text {overlap }}=\int_{0}^{R_{\mathcal{H}}} d^{3} r \Psi_{\psi}(\mathbf{r}) \Psi_{\mathcal{H}}(\mathbf{r})
$$




\begin{tabular}{|ccccc|}
\hline Composition & Quark content & $\alpha_{\psi \psi}(n \ell)\left[\mathrm{GeV}^{-3}\right]$ & $J_{\text {tot }}^{P}$ & Mass (Binding) $[\mathrm{MeV}]$ \\
\hline$\chi_{\mathrm{c} 0}(1 P) \otimes K$ & $n \bar{s} c \bar{c}(s \bar{n} c \bar{c})$ & 11 & $0^{-}$ & $3886(-22)$ \\
$\eta_{\mathrm{c}}(2 S) \otimes K$ & $n \bar{s} c \bar{c}(s \bar{n} c \bar{c})$ & 18 & $0^{+}$ & $3948(-183)$ \\
$\chi_{\mathrm{c} 1}(1 P) \otimes K$ & $n \bar{s} c \bar{c}(s \bar{n} c \bar{c})$ & 11 & $1^{-}$ & $3981(-23)$ \\
$\psi(2 S) \otimes K$ & $n \bar{s} c \bar{c}(s \bar{n} c \bar{c})$ & 18 & $1^{+}$ & $3996(-184)$ \\
$h_{\mathrm{c}}(1 P) \otimes K$ & $n \bar{s} c \bar{c}(s \bar{n} c \bar{c})$ & 11 & $1^{-}$ & $3996(-23)$ \\
$\chi_{\mathrm{c} 2}(1 P) \otimes K$ & $n \bar{s} c \bar{c}(s \bar{n} c \bar{c})$ & 11 & $2^{-}$ & $4027(-23)$ \\
\hline$\chi_{\mathrm{c} 0}(1 P) \otimes K^{*}$ & $n \bar{s} c \bar{c}(s \bar{n} c \bar{c})$ & 11 & $1^{-}$ & $4155(-151)$ \\
$\eta_{\mathrm{c}}(2 S) \otimes K^{*}$ & $n \bar{s} c \bar{c}(s \bar{n} c \bar{c})$ & 18 & $1^{+}$ & $4159(-370)$ \\
$\psi(2 S) \otimes K^{*}$ & $n \bar{s} c \bar{c}(s \bar{n} c \bar{c})$ & 18 & $0^{+}, 1^{+}, 2^{+}$ & $4207(-371)$ \\
$\chi_{\mathrm{c} 1}(1 P) \otimes K^{*}$ & $n \bar{s} c \bar{c}(s \bar{n} c \bar{c})$ & 11 & $0^{-}, 1^{-}, 2^{-}$ & $4250(-152)$ \\
$h_{\mathrm{c}}(1 P) \otimes K^{*}$ & $n \bar{s} c \bar{c}(s \bar{n} c \bar{c})$ & 11 & $0^{-}, 1^{-}, 2^{-}$ & $4265(-153)$ \\
$\chi_{\mathrm{c} 2}(1 P) \otimes K^{*}$ & $n \bar{s} c \bar{c}(s \bar{n} c \bar{c})$ & 11 & $1^{-}, 2^{-}, 3^{-}$ & $4295(-153)$ \\
{$\left[\eta_{\mathrm{c}}(2 S) \otimes K^{*}\right]_{\ell_{\mathrm{hq}}=1}$} & $n \bar{s} c \bar{c}(s \bar{n} c \bar{c})$ & 18 & $0^{-}, 1^{-}, 2^{-}$ & $4436(-93)$ \\
{$\left[\psi(2 S) \otimes K^{*}\right]_{\ell_{\mathrm{hq}}=1}$} & $n \bar{s} c \bar{c}(s \bar{n} c \bar{c})$ & 18 & $0^{-}, 1^{-}, 2^{-}, 3^{-}$ & $4484(-94)$ \\
\hline
\end{tabular}

Table 4. As table 2, but for charmonium- $K$ and $K^{*}(892)$ bound states.

Here, $R_{\mathcal{H}}$ is the light hadron's radius and $\Psi_{\psi}(\mathbf{r})$ and $\Psi_{\mathcal{H}}(\mathbf{r})$ are the wave functions of the heavy and light hadro-quarkonium constituents, respectively, extracted from the relativized QM [76]. If we restrict to the case of kaon-charmonium bound states, the heavy and light hadro-quarkonium's constituents can only be $1 P$ or $2 S$ charmonia (heavy component) and $1 S K$ or $K^{*}$ mesons (light component); see table IV.

We consider two different examples, $\chi_{\mathrm{c} 0}(1 P) \otimes K$ and $\eta_{\mathrm{c}}(2 S) \otimes K$. All the other combinations of heavy and light mesons are analogous to the previous ones, because we expect the radial wave functions of all the other $\chi_{\mathrm{c}}(1 P)$ states to be very similar to that of the $\chi_{\mathrm{c} 0}(1 P)$, namely $\Psi_{\chi_{\mathrm{c} 0}(1 P)}(r) \simeq \Psi_{h_{\mathrm{c}}(1 P)}(r) \simeq \Psi_{\chi_{\mathrm{c} 1}(1 P)}(r) \simeq \Psi_{\chi_{\mathrm{c} 2}(1 P)}(r)$; analogously, we expect that $\Psi_{\psi(2 S)}(r) \simeq \Psi_{\eta_{\mathrm{c}}(2 S)}(r)$ and $\Psi_{K}(r) \simeq \Psi_{K^{*}}(r)$. By calculating the overlap integral of eq. (3.2), we get $P_{\text {overlap }}\left[\chi_{\mathrm{c} 0}(1 P) \otimes K\right]=0$ and $P_{\text {overlap }}\left[\eta_{\mathrm{c}}(2 S) \otimes K\right]=0.01$.

In conclusion, the previous test would indicate that in $\chi_{\mathrm{c} 0}(1 P) \otimes K$ and $\eta_{\mathrm{c}}(2 S) \otimes K$ bound states the approximations we considered are acceptable ones and that there should be no substantial mixing among the heavy and light components.

\section{Relativized diquark model}

We describe tetraquarks as color-antitriplet $\left(\overline{3}_{c}\right)$ diquark and color-triplet $\left(3_{c}\right)$ antidiquark $(\mathcal{D} \overline{\mathcal{D}})$ bound states. We also assume the constituents, $\mathcal{D}$ and $\overline{\mathcal{D}}$, to be inert against internal spatial excitations [83-86]. Consequently, the internal dynamics of the $\mathcal{D} \overline{\mathcal{D}}$ system can be described by means of a single relative coordinate $\mathbf{r}_{\mathrm{rel}}$ with conjugate momentum $\mathbf{q}_{\mathrm{rel}}$. 


\begin{tabular}{|c|c|c|c|c|}
\hline Composition & Quark content & $\alpha_{\psi \psi}(n \ell)\left[\mathrm{GeV}^{-3}\right]$ & $J_{\text {tot }}^{P}$ & Mass (Binding) $[\mathrm{MeV}]$ \\
\hline$\eta_{\mathrm{b}}(2 S) \otimes K$ & $n \bar{s} b \bar{b}(s \bar{n} b \bar{b})$ & 23 & $0^{+}$ & $10121(-372)$ \\
\hline$\Upsilon(2 S) \otimes K$ & $n \bar{s} b \bar{b}(s \bar{n} b \bar{b})$ & 23 & $1^{+}$ & $10145(-372)$ \\
\hline$\chi_{\mathrm{b} 0}(1 P) \otimes K$ & $n \bar{s} b \bar{b}(s \bar{n} b \bar{b})$ & 14 & $0^{-}$ & $10254(-99)$ \\
\hline$\chi_{\mathrm{b} 1}(1 P) \otimes K$ & $n \bar{s} b \bar{b}(s \bar{n} b \bar{b})$ & 14 & $1^{-}$ & $10288(-99)$ \\
\hline$h_{\mathrm{b}}(1 P) \otimes K$ & $n \bar{s} b \bar{b}(s \bar{n} b \bar{b})$ & 14 & $1^{-}$ & $10294(-99)$ \\
\hline$\chi_{\mathrm{b} 2}(1 P) \otimes K$ & $n \bar{s} b \bar{b}(s \bar{n} b \bar{b})$ & 14 & $2^{-}$ & $10307(-99)$ \\
\hline$\eta_{\mathrm{b}}(2 S) \otimes K$ & $n \bar{s} b \bar{b}(s \bar{n} b \bar{b})$ & 33 & $0^{+}$ & $9755(-738)$ \\
\hline$\Upsilon(2 S) \otimes K$ & $n \bar{s} b \bar{b}(s \bar{n} b \bar{b})$ & 33 & $1^{+}$ & $9779(-738)$ \\
\hline$\chi_{\mathrm{b} 0}(1 P) \otimes K$ & $n \bar{s} b \bar{b}(s \bar{n} b \bar{b})$ & 21 & $0^{-}$ & $10049(-305)$ \\
\hline$\chi_{\mathrm{b} 1}(1 P) \otimes K$ & $n \bar{s} b \bar{b}(s \bar{n} b \bar{b})$ & 21 & $1^{-}$ & $10082(-305)$ \\
\hline$h_{\mathrm{b}}(1 P) \otimes K$ & $n \bar{s} b \bar{b}(s \bar{n} b \bar{b})$ & 21 & $1^{-}$ & $10088(-305)$ \\
\hline$\chi_{\mathrm{b} 2}(1 P) \otimes K$ & $n \bar{s} b \bar{b}(s \bar{n} b \bar{b})$ & 21 & $2^{-}$ & $10101(-305)$ \\
\hline$\left[\eta_{\mathrm{b}}(2 S) \otimes K\right]_{\ell_{\mathrm{hq}}=1}$ & $n \bar{s} b \bar{b}(s \bar{n} b \bar{b})$ & 33 & $1^{-}$ & $10425(-68)$ \\
\hline$[\Upsilon(2 S) \otimes K]_{\ell_{\mathrm{hq}}=1}$ & $n \bar{s} b \bar{b}(s \bar{n} b \bar{b})$ & 33 & $0^{-}, 1^{-}$or $2^{-}$ & $10449(-68)$ \\
\hline$\eta_{\mathrm{b}}(2 S) \otimes K^{*}$ & $n \bar{s} b \bar{b}(s \bar{n} b \bar{b})$ & 23 & $1^{+}$ & $10323(-568)$ \\
\hline$\Upsilon(2 S) \otimes K^{*}$ & $n \bar{s} b \bar{b}(s \bar{n} b \bar{b})$ & 23 & $0^{+}, 1^{+}, 2^{+}$ & $10347(-568)$ \\
\hline$\chi_{\mathrm{b} 0}(1 P) \otimes K^{*}$ & $n \bar{s} b \bar{b}(s \bar{n} b \bar{b})$ & 14 & $1^{-}$ & $10484(-267)$ \\
\hline$\chi_{\mathrm{b} 1}(1 P) \otimes K^{*}$ & $n \bar{s} b \bar{b}(s \bar{n} b \bar{b})$ & 14 & $0^{-}, 1^{-}, 2^{-}$ & $10517(-267)$ \\
\hline$h_{\mathrm{b}}(1 P) \otimes K^{*}$ & $n \bar{s} b \bar{b}(s \bar{n} b \bar{b})$ & 14 & $0^{-}, 1^{-}, 2^{-}$ & $10524(-267)$ \\
\hline$\chi_{\mathrm{b} 2}(1 P) \otimes K^{*}$ & $n \bar{s} b \bar{b}(s \bar{n} b \bar{b})$ & 14 & $1^{-}, 2^{-}, 3^{-}$ & $10536(-267)$ \\
\hline$\left[\eta_{\mathrm{b}}(2 S) \otimes K^{*}\right]_{\ell_{\mathrm{hq}}=1}$ & $n \bar{s} b \bar{b}(s \bar{n} b \bar{b})$ & 23 & $0^{-}, 1^{-}, 2^{-}$ & $10604(-286)$ \\
\hline$\left[\Upsilon(2 S) \otimes K^{*}\right]_{\ell_{\mathrm{hq}}=1}$ & $n \bar{s} b \bar{b}(s \bar{n} b \bar{b})$ & 23 & $0^{-}, 1^{-}, 2^{-}, 3^{-}$ & $10629(-286)$ \\
\hline$\left[\chi_{\mathrm{b} 0}(1 P) \otimes K^{*}\right]_{\ell_{\mathrm{hq}}=1}$ & $n \bar{s} b \bar{b}(s \bar{n} b \bar{b})$ & 14 & $0^{+}, 1^{+}, 2^{+}$ & $10711(-40)$ \\
\hline$\left[\chi_{\mathrm{b} 1}(1 P) \otimes K^{*}\right]_{\ell_{\mathrm{hq}}=1}$ & $n \bar{s} b \bar{b}(s \bar{n} b \bar{b})$ & 14 & $0^{+}, 1^{+}, 2^{+}, 3^{+}$ & $10745(-40)$ \\
\hline$\left[h_{\mathrm{b}}(1 P) \otimes K^{*}\right]_{\ell_{\mathrm{hq}}=1}$ & $n \bar{s} b \bar{b}(s \bar{n} b \bar{b})$ & 14 & $0^{+}, 1^{+}, 2^{+}, 3^{+}$ & $10751(-40)$ \\
\hline$\left[\chi_{\mathrm{b} 2}(1 P) \otimes K^{*}\right]_{\ell_{\mathrm{hq}}=1}$ & $n \bar{s} b \bar{b}(s \bar{n} b \bar{b})$ & 14 & $0^{+}, 1^{+}, 2^{+}, 3^{+}, 4^{+}$ & $10764(-40)$ \\
\hline$\eta_{\mathrm{b}}(2 S) \otimes K^{*}$ & $n \bar{s} b \bar{b}(s \bar{n} b \bar{b})$ & 33 & $1^{+}$ & $9974(-917) ; 10786(-105)$ \\
\hline$\Upsilon(2 S) \otimes K^{*}$ & $n \bar{s} b \bar{b}(s \bar{n} b \bar{b})$ & 33 & $0^{+}, 1^{+}, 2^{+}$ & $9998(-917) ; 10810(-105)$ \\
\hline$\chi_{\mathrm{b} 0}(1 P) \otimes K^{*}$ & $n \bar{s} b \bar{b}(s \bar{n} b \bar{b})$ & 21 & $1^{-}$ & $10252(-499)$ \\
\hline$\left[\eta_{\mathrm{b}}(2 S) \otimes K^{*}\right]_{\ell_{\mathrm{hq}}=1}$ & $n \bar{s} b \bar{b}(s \bar{n} b \bar{b})$ & 33 & $0^{-}, 1^{-}, 2^{-}$ & $10284(-607)$ \\
\hline$\chi_{\mathrm{b} 1}(1 P) \otimes K^{*}$ & $n \bar{s} b \bar{b}(s \bar{n} b \bar{b})$ & 21 & $0^{-}, 1^{-}, 2^{-}$ & $10285(-499)$ \\
\hline$h_{\mathrm{b}}(1 P) \otimes K^{*}$ & $n \bar{s} b \bar{b}(s \bar{n} b \bar{b})$ & 21 & $0^{-}, 1^{-}, 2^{-}$ & $10292(-499)$ \\
\hline$\chi_{\mathrm{b} 2}(1 P) \otimes K^{*}$ & $n \bar{s} b \bar{b}(s \bar{n} b \bar{b})$ & 21 & $1^{-}, 2^{-}, 3^{-}$ & $10305(-499)$ \\
\hline$\left[\Upsilon(2 S) \otimes K^{*}\right]_{\ell_{\mathrm{hq}}=1}$ & $n \bar{s} b \bar{b}(s \bar{n} b \bar{b})$ & 33 & $0^{-}, 1^{-}, 2^{-}, 3^{-}$ & $10308(-607)$ \\
\hline$\left[\chi_{\mathrm{b} 0}(1 P) \otimes K^{*}\right]_{\ell_{\mathrm{hq}}=1}$ & $n \bar{s} b \bar{b}(s \bar{n} b \bar{b})$ & 21 & $0^{+}, 1^{+}, 2^{+}$ & $10525(-226)$ \\
\hline$\left[\chi_{\mathrm{b} 1}(1 P) \otimes K^{*}\right]_{\ell_{\mathrm{hq}}=1}$ & $n \bar{s} b \bar{b}(s \bar{n} b \bar{b})$ & 21 & $0^{+}, 1^{+}, 2^{+}, 3^{+}$ & $10558(-226)$ \\
\hline$\left[h_{\mathrm{b}}(1 P) \otimes K^{*}\right]_{\ell_{\mathrm{hq}}=1}$ & $n \bar{s} b \bar{b}(s \bar{n} b \bar{b})$ & 21 & $0^{+}, 1^{+}, 2^{+}, 3^{+}$ & $10565(-226)$ \\
\hline$\left[\chi_{\mathrm{b} 2}(1 P) \otimes K^{*}\right]_{\ell_{\mathrm{hq}}=1}$ & $n \bar{s} b \bar{b}(s \bar{n} b \bar{b})$ & 21 & $0^{+}, 1^{+}, 2^{+}, 3^{+}, 4^{+}$ & $10578(-226)$ \\
\hline
\end{tabular}

Table 5. As table 2, but for bottomonium- $K$ and $K^{*}(892)$ bound states. 
The Hamiltonian of the system is given by $[46,48,50]$

$$
\begin{aligned}
\mathcal{H}^{\mathrm{REL}} & =T+V\left(\mathbf{r}_{\mathrm{rel}}\right), \\
T & =\sqrt{\mathbf{q}_{\mathrm{rel}}^{2}+m_{\mathcal{D}_{a}}^{2}}+\sqrt{\mathbf{q}_{\mathrm{rel}}^{2}+m_{\overline{\mathcal{D}}_{b}}^{2}},
\end{aligned}
$$

where the potential

$$
\begin{aligned}
V\left(r_{\text {rel }}\right)= & \beta r_{\text {rel }}+G\left(r_{\text {rel }}\right)+\frac{2 \mathbf{S}_{D_{a}} \cdot \mathbf{S}_{\bar{D}_{b}}}{3 m_{\mathcal{D}_{a}} m_{\overline{\mathcal{D}}_{b}}} \nabla^{2} G\left(r_{\text {rel }}\right)-\frac{1}{3 m_{\mathcal{D}_{a}} m_{\overline{\mathcal{D}}_{b}}}\left(3 \mathbf{S}_{\mathcal{D}_{a}} \cdot \hat{r}_{\text {rel }} \mathbf{S}_{\overline{\mathcal{D}}_{b}} \cdot \hat{r}_{\text {rel }}-\mathbf{S}_{\mathcal{D}_{a}} \cdot \mathbf{S}_{\overline{\mathcal{D}}_{b}}\right) \\
& \times\left(\frac{\partial^{2}}{\partial r_{\text {rel }}^{2}}-\frac{1}{r_{\text {rel }}} \frac{\partial}{\partial r_{\text {rel }}}\right) G\left(r_{\text {rel }}\right)+\Delta E
\end{aligned}
$$

is the sum of linear-confinement and one-gluon exchange (OGE) terms [48, 76, 87, 88]. The Coulomb-like part is $[76,88]$

$$
G\left(r_{\mathrm{rel}}\right)=-\frac{4 \alpha_{\mathrm{s}}\left(r_{\mathrm{rel}}\right)}{3 r_{\mathrm{rel}}}=-\sum_{k} \frac{4 \alpha_{k}}{3 r_{\mathrm{rel}}} \operatorname{Erf}\left(\tau_{\mathcal{D}_{a} \overline{\mathcal{D}}_{b} k} r_{\mathrm{rel}}\right)
$$

where Erf is the error function and $[76,88]$

$$
\tau_{\mathcal{D}_{a} \overline{\mathcal{D}}_{b} k}=\frac{\gamma_{k} \sigma_{\mathcal{D}_{a} \overline{\mathcal{D}}_{b}}}{\sqrt{\sigma_{\mathcal{D}_{a} \overline{\mathcal{D}}_{b}}^{2}+\gamma_{k}^{2}}} ; \quad \sigma_{\mathcal{D}_{a} \overline{\mathcal{D}}_{b}}=\sqrt{\frac{1}{2} \sigma_{0}^{2}\left[1+\left(\frac{4 m_{\mathcal{D}_{a}} m_{\overline{\mathcal{D}}_{b}}}{\left(m_{\mathcal{D}_{a}}+m_{\overline{\mathcal{D}}_{b}}\right)^{2}}\right)^{4}\right]+s^{2}\left(\frac{2 m_{\mathcal{D}_{a}} m_{\overline{\mathcal{D}}_{b}}}{m_{\mathcal{D}_{a}}+m_{\overline{\mathcal{D}}_{b}}}\right)^{2}} .
$$

The model parameters are listed in table 6 . The strength of the linear confining interaction, $\beta$, and the value of the constant, $\Delta E$, in eq. (4.2) are taken from [48, table I]; the values of the parameters $\alpha_{k}$ and $\gamma_{k}(k=1,2,3), \sigma_{0}$ and $s$ are extracted from refs. [76, 88]. The masses of the scalar and axial-vector diquarks $c n, c s, b n$ and $b s$, are taken from refs. [48, 50, 89].

Therefore, the results we report below are parameter-free predictions. The present model was previously used to calculate the spectrum of hidden-charm [48] and fully-heavy tetraquarks $[46,50]$.

\section{Masses of $c s \bar{c} \bar{n}$ and $b s \bar{b} \bar{n}$ states in the compact tetraquark model}

Below, we provide results for the ground-state masses and the spectrum of strange hiddencharm $(c s \bar{c} \bar{n}$ and $c n \bar{c} \bar{s})$ and bottom $(b s \bar{b} \bar{n}$ and $b n \bar{b} \bar{s})$ tetraquarks in the compact tetraquark model of refs. [46, 48, 50] and section 4 .

The tetraquark masses are obtained by solving the eigenvalue problem of eq. (4.1) by means of a numerical variational procedure, based on harmonic oscillator trial wave functions. This variational method was previously applied to meson and baryon spectroscopy $[46,48,50,85,86]$.

\subsection{Ground-state energies of $c s \bar{c} \bar{n}$ and $b s \bar{b} \bar{n}$ tetraquarks}

Our starting point is the calculation of the ground-state masses of $c s \bar{c} \bar{n}(c n \bar{c} \bar{s})$ and $b s \bar{b} \bar{n}$ $(b n \bar{b} \bar{s})$ tetraquark configurations. 


\begin{tabular}{|cccc|}
\hline Parameter & Value & Parameter & Value \\
\hline$\alpha_{1}$ & 0.25 & $\gamma_{1}$ & $2.53 \mathrm{fm}^{-1}$ \\
$\alpha_{2}$ & 0.15 & $\gamma_{2}$ & $8.01 \mathrm{fm}^{-1}$ \\
$\alpha_{3}$ & 0.20 & $\gamma_{3}$ & $80.1 \mathrm{fm}^{-1}$ \\
$\sigma_{0}$ & $9.29 \mathrm{fm}^{-1}$ & $s$ & 1.55 \\
$\beta$ & $3.90 \mathrm{fm}^{-2}$ & $\Delta E$ & $-370 \mathrm{MeV}$ \\
$M_{c n}^{\mathrm{sc}}$ & $1933 \mathrm{MeV}$ & $M_{c n}^{\mathrm{av}}$ & $2250 \mathrm{MeV}$ \\
$M_{c s}^{\mathrm{sc}}$ & $2229 \mathrm{MeV}$ & $M_{c s}^{\mathrm{av}}$ & $2264 \mathrm{MeV}$ \\
$M_{b n}^{\mathrm{sc}}$ & $5451 \mathrm{MeV}$ & $M_{b n}^{\mathrm{av}}$ & $5465 \mathrm{MeV}$ \\
$M_{b s}^{\mathrm{sc}}$ & $5572 \mathrm{MeV}$ & $M_{b s}^{\text {av }}$ & $5585 \mathrm{MeV}$ \\
\hline
\end{tabular}

Table 6. Parameters of the Hamiltonian (4.1). Here $n=u$ or $d$ and the superscripts "sc" and "av" indicate scalar and axial-vector diquarks, respectively.

In the first case, we obtain

$$
M_{c s \bar{n} \bar{n}}^{\mathrm{gs}}=\left\{\begin{array}{ll}
3.85 \mathrm{GeV} & \text { (sc-sc configuration) } \\
3.66 \mathrm{GeV} & (\text { av-av configuration })
\end{array},\right.
$$

where the notations "sc" and "av" indicate scalar and axial-vector diquarks, respectively. The previous values have be compared with the experimental energy of the $D \bar{D}_{\mathrm{s}}$ threshold, $3.84 \mathrm{GeV}$ [74]. It is interesting to observe that the av-av $c s \bar{c} \bar{n}$ tetraquark ground-state is around $200 \mathrm{MeV}$ below the lowest energy hadro-charmonium $\eta_{\mathrm{c}} \otimes K$ state of table 4 , which lies at an energy of $3886 \mathrm{MeV}$.

In the second case, we get

$$
M_{b s \bar{b} \bar{n}}^{\mathrm{gs}}=\left\{\begin{array}{ll}
10.41 \mathrm{GeV} & (\text { sc-sc configuration }) \\
10.23 \mathrm{GeV} & (\text { av-av configuration })
\end{array},\right.
$$

to be compared with the $B \bar{B}_{\mathrm{s}}$ threshold energy, $10.65 \mathrm{GeV}$ [74]. Contrary to the $\operatorname{cs} \bar{c} \bar{n}$ case, the av-av $b s \bar{b} \bar{n}$ tetraquark ground-state is above the lowest energy hadro-bottomonium $\eta_{\mathrm{b}} \otimes K$ state of table 5 , which lies at an energy between 9.76 and $10.12 \mathrm{GeV}$ depending on the input value of the chromo-electric polarizability.

According to the previous results, strange hidden-charm and bottom tetraquarks may be bound. However, due to the largeness of the theoretical uncertainties on the $b s \bar{b} \bar{n}$ and, especially, $c s \bar{c} \bar{n}$ ground-state tetraquark masses, it is difficult to draw a definitive conclusion.

\subsection{Spectra of $c s \bar{c} \bar{n}$ and $b s \bar{b} \bar{n}$ tetraquarks}

After discussing the possible emergence of $c s \bar{c} \bar{n}$ and $b s \bar{b} \bar{n}$ tetraquarks and their groundstate energies, the next step is to calculate the spectrum predicted by the Hamiltonian of eq. (4.1) with the model parameters of table 6 . In tables 7 and 8 we report the masses of $c s \bar{c} \bar{n}(c n \bar{c} \bar{s})$ and $b s \bar{b} \bar{n}(b n \bar{b} \bar{s})$ compact tetraquarks, where $n=u$ or $d$, up to the second radial excitations. 


\begin{tabular}{|c|c|c|c|c|c|c|c|c|}
\hline \multicolumn{9}{|c|}{$\operatorname{cs} \overline{\mathbf{c}} \overline{\mathbf{n}}$} \\
\hline$J^{P C}$ & $N\left[\left(S_{D}, S_{\bar{D}}\right) S, L\right] J$ & $E^{\text {th }}[\mathrm{MeV}]$ & $J^{P C}$ & $N\left[\left(S_{D}, S_{\bar{D}}\right) S, L\right] J$ & $E^{\text {th }}[\mathrm{MeV}]$ & $J^{P C}$ & $N\left[\left(S_{D}, S_{\bar{D}}\right) S, L\right] J$ & $E^{\text {th }}[\mathrm{MeV}]$ \\
\hline $0^{++}$ & $1[(1,1) 0,0] 0$ & 3657 & $1^{++}$ & $1[(1,0) 1,0] 1$ & 4016 & $0^{-+}$ & $1[(1,0) 1,1] 0$ & 4396 \\
\hline $0^{++}$ & $1[(0,0) 0,0] 0$ & 3852 & $1^{++}$ & $2[(1,0) 1,0] 1$ & 4544 & $0^{-+}$ & $1[(1,1) 1,1] 0$ & 4580 \\
\hline $0^{++}$ & $2[(0,0) 0,0] 0$ & 4383 & $1^{++}$ & $1[(1,0) 1,2] 1$ & 4658 & $0^{-+}$ & $2[(1,0) 1,1] 0$ & 4783 \\
\hline $0^{++}$ & $2[(1,1) 0,0] 0$ & 4496 & $1^{++}$ & $1[(1,1) 2,2] 1$ & 4825 & $0^{-+}$ & $2[(1,1) 1,1] 0$ & 4960 \\
\hline $0^{++}$ & $3[(0,0) 0,0] 0$ & 4747 & $1^{++}$ & $3[(1,0) 1,0] 1$ & 4906 & $0^{-+}$ & $3[(1,0) 1,1] 0$ & 5093 \\
\hline $0^{++}$ & $1[(1,1) 2,2] 0$ & 4830 & $1^{++}$ & $2[(1,0) 1,2] 1$ & 4982 & $0^{-+}$ & $3[(1,1) 1,1] 0$ & 5265 \\
\hline $0^{++}$ & $3[(1,1) 0,0] 0$ & 4913 & $1^{++}$ & $2[(1,1) 2,2] 1$ & 5147 & & & \\
\hline $0^{++}$ & $2[(1,1) 2,2] 0$ & 5151 & $1^{++}$ & $3[(1,0) 1,2] 1$ & 5261 & & & \\
\hline $0^{++}$ & $3[(1,1) 2,2] 0$ & 5427 & $1^{++}$ & $3[(1,1) 2,2] 1$ & 5423 & & & \\
\hline $1^{--}$ & $1[(0,0) 0,1] 1$ & 4234 & $2^{++}$ & $1[(1,1) 2,0] 2$ & 4232 & $1^{+-}$ & $1[(1,0) 1,0] 1$ & 4016 \\
\hline $1^{--}$ & $1[(1,0) 1,1] 1$ & 4396 & $2^{++}$ & $1[(0,0) 0,2] 2$ & 4497 & $1^{+-}$ & $1[(1,1) 1,0] 1$ & 4061 \\
\hline $1^{--}$ & $1[(1,1) 0,1] 1$ & 4558 & $2^{++}$ & $2[(1,1) 2,0] 2$ & 4739 & $1^{+-}$ & $2[(1,0) 1,0] 1$ & 4544 \\
\hline $1^{--}$ & $1[(1,1) 2,1] 1$ & 4583 & $2^{++}$ & $1[(1,1) 2,2] 2$ & 4818 & $1^{+-}$ & $2[(1,1) 1,0] 1$ & 4637 \\
\hline $1^{--}$ & $2[(0,0) 0,1] 1$ & 4622 & $2^{++}$ & $1[(1,1) 0,2] 2$ & 4819 & $1^{+-}$ & $1[(1,0) 1,2] 1$ & 4658 \\
\hline $1^{--}$ & $2[(1,0) 1,1] 1$ & 4783 & $2^{++}$ & $2[(0,0) 0,2] 2$ & 4824 & $1^{+-}$ & $1[(1,1) 1,2] 1$ & 4822 \\
\hline $1^{--}$ & $2[(1,1) 0,1] 1$ & 4942 & $2^{++}$ & $3[(1,1) 2,0] 2$ & 5092 & $1^{+-}$ & $3[(1,0) 1,0] 1$ & 4906 \\
\hline $1^{--}$ & $2[(1,1) 2,1] 1$ & 4962 & $2^{++}$ & $3[(0,0) 0,2] 2$ & 5105 & $1^{+-}$ & $2[(1,0) 1,2] 1$ & 4982 \\
\hline $1^{--}$ & $3[(0,0) 0,1] 1$ & 4935 & $2^{++}$ & $2[(1,1) 0,2] 2$ & 5140 & $1^{+-}$ & $3[(1,1) 1,0] 1$ & 5010 \\
\hline $1^{--}$ & $3[(1,0) 1,1] 1$ & 5093 & $2^{++}$ & $2[(1,1) 2,2] 2$ & 5140 & $1^{+-}$ & $2[(1,1) 1,2] 1$ & 5144 \\
\hline $1^{--}$ & $3[(1,1) 0,1] 1$ & 5250 & $2^{++}$ & $3[(1,1) 0,2] 2$ & 5416 & $1^{+-}$ & $3[(1,0) 1,2] 1$ & 5261 \\
\hline $1^{--}$ & $3[(1,1) 2,1] 1$ & 5268 & $2^{++}$ & $3[(1,1) 2,2] 2$ & 5416 & $1^{+-}$ & $3[(1,1) 1,2] 1$ & 5420 \\
\hline $0^{--}$ & $1[(1,0) 1,1] 0$ & 4396 & & & & & & \\
\hline $0^{--}$ & $2[(1,0) 1,1] 0$ & 4783 & & & & & & \\
\hline $0^{--}$ & $3[(1,0) 1,1] 0$ & 5093 & & & & & & \\
\hline
\end{tabular}

Table 7. Masses of $c s \bar{c} \bar{n}(c n \bar{c} \bar{s})$ tetraquarks, obtained by solving the eigenvalue problem of eq. (4.1). We report states up to the second radial excitation. They are labelled thus: $N$ is the radial quantum number; $S_{\mathrm{D}}, S_{\overline{\mathrm{D}}}$ are the spin of the diquark and antidiquark, respectively, coupled to the total spin of the meson, $S$; the latter is coupled to the orbital angular momentum, $L$, to get the total angular momentum of the tetraquark, $J$. In the case of scalar-axial-vector diquark configurations, there are two possible ways of combining diquarks to get a tetraquark with strangeness $\mathcal{S}= \pm 1$. They are $[c n]\{\bar{c} \bar{s}\}$ and $[c s]\{\bar{c} \bar{n}\}$, where the diquarks in square brackets are scalar and those in curly brackets are axial-vector. In these cases, the values of the tetraquark masses shown are the average of the energies corresponding to the previous $[c n]\{\bar{c} \bar{s}\}$ and $[c s]\{\bar{c} \bar{n}\}$ spin-flavor configurations.

As discussed in ref. [50], these type of predictions may serve as benchmarks for other analyses with the goal of identifying model-dependent artifacts and develop a perspective on those predictions which might only be weakly sensitive to model details. Moreover, given the possibility that $J=0^{++}$tetraquarks may be more difficult to access experimentally than $1^{--}$resonances, our predictions for $J \neq 0$ states may be useful in the experimental search for $c s \bar{c} \bar{n}$ and $b s \bar{b} \bar{n}$ tetraquark states.

The calculation of the spectrum is only the first step of a wider analysis, with the aim of understanding the possible formation and stability of compact tetraquark states. The 


\begin{tabular}{|c|c|c|c|c|c|c|c|c|}
\hline \multicolumn{9}{|c|}{ bs $\bar{b} \overline{\mathbf{n}}$} \\
\hline$J^{P C}$ & $N\left[\left(S_{D}, S_{\bar{D}}\right) S, L\right] J$ & $E^{\text {th }}[\mathrm{MeV}]$ & $J^{P C}$ & $N\left[\left(S_{D}, S_{\bar{D}}\right) S, L\right] J$ & $E^{\text {th }}[\mathrm{MeV}]$ & $J^{P C}$ & $N\left[\left(S_{D}, S_{\bar{D}}\right) S, L\right] J$ & $E^{\text {th }}[\mathrm{MeV}]$ \\
\hline $0^{++}$ & $1[(1,1) 0,0] 0$ & 10234 & $1^{++}$ & $1[(1,0) 1,0] 1$ & 10420 & $0^{-+}$ & $1[(1,0) 1,1] 0$ & 10804 \\
\hline $0^{++}$ & $1[(0,0) 0,0] 0$ & 10407 & $1^{++}$ & $2[(1,0) 1,0] 1$ & 10922 & $0^{-+}$ & $1[(1,1) 1,1] 0$ & 10827 \\
\hline $0^{++}$ & $2[(1,1) 0,0] 0$ & 10848 & $1^{++}$ & $1[(1,0) 1,2] 1$ & 11039 & $0^{-+}$ & $2[(1,0) 1,1] 0$ & 11139 \\
\hline $0^{++}$ & $2[(0,0) 0,0] 0$ & 10909 & $1^{++}$ & $1[(1,1) 2,2] 1$ & 11054 & $0^{-+}$ & $2[(1,1) 1,1] 0$ & 11159 \\
\hline $0^{++}$ & $1[(1,1) 2,2] 0$ & 11056 & $1^{++}$ & $3[(1,0) 1,0] 1$ & 11235 & $0^{-+}$ & $3[(1,0) 1,1] 0$ & 11398 \\
\hline $0^{++}$ & $3[(1,1) 0,0] 0$ & 11185 & $1^{++}$ & $2[(1,0) 1,2] 1$ & 11311 & $0^{-+}$ & $3[(1,1) 1,1] 0$ & 11418 \\
\hline $0^{++}$ & $3[(0,0) 0,0] 0$ & 11222 & $1^{++}$ & $2[(1,1) 2,2] 1$ & 11326 & & & \\
\hline $0^{++}$ & $2[(1,1) 2,2] 0$ & 11328 & $1^{++}$ & $3[(1,0) 1,2] 1$ & 11539 & & & \\
\hline $0^{++}$ & $3[(1,1) 2,2] 0$ & 11556 & $1^{++}$ & $3[(1,1) 2,2] 1$ & 11554 & & & \\
\hline $1^{--}$ & $1[(0,0) 0,1] 1$ & 10790 & $2^{++}$ & $1[(1,1) 2,0] 2$ & 10467 & $1^{+-}$ & $1[(1,1) 1,0] 1$ & 10373 \\
\hline $1^{--}$ & $1[(1,0) 1,1] 1$ & 10804 & $2^{++}$ & $2[(1,1) 2,0] 2$ & 10952 & $1^{+-}$ & $1[(1,0) 1,0] 1$ & 10420 \\
\hline $1^{--}$ & $1[(1,1) 0,1] 1$ & 10816 & $2^{++}$ & $1[(0,0) 0,2] 2$ & 11025 & $1^{+-}$ & $2[(1,1) 1,0] 1$ & 10906 \\
\hline $1^{--}$ & $1[(1,1) 2,1] 1$ & 10828 & $2^{++}$ & $1[(1,1) 0,2] 2$ & 11051 & $1^{+-}$ & $2[(1,0) 1,0] 1$ & 10922 \\
\hline $1^{--}$ & $2[(0,0) 0,1] 1$ & 11125 & $2^{++}$ & $1[(1,1) 2,2] 2$ & 11051 & $1^{+-}$ & $1[(1,0) 1,2] 1$ & 11039 \\
\hline $1^{--}$ & $2[(1,0) 1,1] 1$ & 11139 & $2^{++}$ & $3[(1,1) 2,0] 2$ & 11261 & $1^{+-}$ & $1[(1,1) 1,2] 1$ & 11053 \\
\hline $1^{--}$ & $2[(1,1) 0,1] 1$ & 11151 & $2^{++}$ & $2[(0,0) 0,2] 2$ & 11297 & $1^{+-}$ & $3[(1,1) 1,0] 1$ & 11226 \\
\hline $1^{--}$ & $2[(1,1) 2,1] 1$ & 11160 & $2^{++}$ & $2[(1,1) 0,2] 2$ & 11323 & $1^{+-}$ & $3[(1,0) 1,0] 1$ & 11235 \\
\hline $1^{--}$ & $3[(0,0) 0,1] 1$ & 11385 & $2^{++}$ & $2[(1,1) 2,2] 2$ & 11323 & $1^{+-}$ & $2[(1,0) 1,2] 1$ & 11311 \\
\hline $1^{--}$ & $3[(1,0) 1,1] 1$ & 11398 & $2^{++}$ & $3[(0,0) 0,2] 2$ & 11526 & $1^{+-}$ & $2[(1,1) 1,2] 1$ & 11325 \\
\hline $1^{--}$ & $3[(1,1) 0,1] 1$ & 11411 & $2^{++}$ & $3[(1,1) 0,2] 2$ & 11552 & $1^{+-}$ & $3[(1,0) 1,2] 1$ & 11539 \\
\hline $1^{--}$ & $3[(1,1) 2,1] 1$ & 11418 & $2^{++}$ & $3[(1,1) 2,2] 2$ & 11552 & $1^{+-}$ & $3[(1,1) 1,2] 1$ & 11553 \\
\hline $0^{--}$ & $1[(1,0) 1,1] 0$ & 10804 & & & & & & \\
\hline $0^{--}$ & $2[(1,0) 1,1] 0$ & 11139 & & & & & & \\
\hline $0^{--}$ & $3[(1,0) 1,1] 0$ & 11398 & & & & & & \\
\hline
\end{tabular}

Table 8. As table 7, but for $b s \bar{b} \bar{n}(b n \bar{b} \bar{s})$ states.

following steps include the calculation of tetraquark decay amplitudes, production crosssections, and the study of their production mechanisms. When compared to the same observables calculated within other interpretations for $X Y Z$ states (like the meson-meson molecular model, the hadro-quarkonium model and the UQM) and the experimental data, it will be possible to distinguish among the different interpretations and possibly rule out those which are not compatible with the experimental resuls.

In conclusion, even though the experimental search for strange hidden-charm and bottom tetraquarks may be challenging, the observation of these systems may be extremely useful to understand the quark structure of $X Y Z$ exotic mesons.

\section{Conclusion}

We calculated the spectrum of strange hidden-charm and bottom tetraquarks both in the hadro-quarkonium model of refs. [53, 64, 65] and the compact tetraquark model of refs. $[46,48,50]$. We also computed that of hidden-charm and bottom pentaquarks in the hadro-quarkonium model. In particular, we discussed the possible emergence of $\eta_{\mathrm{b}, \mathrm{c}}(2 S)$-, 
$\psi(2 S)-, \Upsilon(2 S)$-, and $\chi_{\mathrm{b}, \mathrm{c}}(1 P)$-hyperon/kaon bound states and the possible formation of $c s \bar{c} \bar{n}$ and $b s \bar{b} \bar{n}$ tetraquarks as diquark-antidiquark bound states.

Our results suggest that: I) strange hadro-quarkonium systems may be strongly bound. On the other hand, if the heavy quarkonium- $(\psi)$ light hadron $(\mathcal{H})$ binding energies become too large, the hadro-quarkonium picture may break down. As a consequence, the $\psi$ and $\mathcal{H}$ components may overlap, and a compact four/five-quark system could be realized rather than a $\psi-\mathcal{H}$ bound state; II) both $c s \bar{c} \bar{n}$ and $b s \bar{b} \bar{n}$ compact tetraquarks may be bound, even though $b s \bar{b} \bar{n}$ configurations are more likely to manifest; III) in the case of $\operatorname{cs} \bar{c} \bar{n}$ configurations, the compact tetraquark ground-state is around $200 \mathrm{MeV}$ below the lowest energy hadro-charmonium state, $\eta_{\mathrm{c}} \otimes K$. On the contrary, in the $b s \bar{b} \bar{n}$ case the compact tetraquark ground-state is above the lowest energy hadro-bottomonium configuration, $\eta_{\mathrm{b}} \otimes K$; IV) by combining the conclusions discussed at points I) and II), we suggest the experimentalists to look for strange tetra- and pentaquark configurations with hidden-bottom. They should be more stable than their hidden-charm counterparts due to kinetic energy suppression; thus, there is a higher probability of observing them.

Finally, as pointed out in ref. [13], the meson-meson molecular model cannot be used to describe heavy-light tetraquarks with non-null strangeness content. The reason is that one-pion-exchange cannot take place between strange and nonstrange heavy mesons, like $B$ and $B_{\mathrm{s}}$. Hidden-charm and bottom mesons with strangeness are also forbidden in the context of the Unquenched Quark Model (UQM) formalism. Indeed, one cannot dress heavy quarkonium $Q \bar{Q}$ states with $Q \bar{s}-n \bar{Q}$ or $Q \bar{n}-s \bar{Q}$ higher Fock components (where $n=u$ or $d$ ) by creating a light $n \bar{n}$ or $s \bar{s}$ pair with vacuum quantum numbers. Tetraquarks with non-null strangeness content can only take place either in the compact tetraquark or hadroquarkonium models. Therefore, a possible way to discriminate between the compact tetraquark and hadro-quarkonium models on one side and the molecular model and UQM interpretations on the other is the experimental search for strange hidden-charm and bottom four-quark states.

Our predictions for $P_{\mathrm{c}}$ and $P_{\mathrm{b}}$ pentaquarks with non-null strangeness content may be soon be tested by $\mathrm{LHCb}$.

\section{Acknowledgments}

This work was supported by the U.S. Department of Energy (Grant No. DE-FG-02-91ER40608) and the Academy of Finland, Project No. 320062. 
Open Access. This article is distributed under the terms of the Creative Commons Attribution License (CC-BY 4.0), which permits any use, distribution and reproduction in any medium, provided the original author(s) and source are credited.

\section{References}

[1] Belle collaboration, Observation of a narrow charmonium-like state in exclusive $B^{ \pm} \rightarrow K^{ \pm} \pi^{+} \pi^{-} J / \psi$ decays, Phys. Rev. Lett. 91 (2003) 262001 [hep-ex/0309032] [INSPIRE].

[2] CDF collaboration, Observation of the narrow state $X(3872) \rightarrow J / \psi \pi^{+} \pi^{-}$in $\bar{p} p$ collisions at $\sqrt{s}=1.96 \mathrm{TeV}$, Phys. Rev. Lett. 93 (2004) 072001 [hep-ex/0312021] [INSPIRE].

[3] D0 collaboration, Observation and properties of the $X(3872)$ decaying to $J / \psi \pi^{+} \pi^{-}$in $p \bar{p}$ collisions at $\sqrt{s}=1.96 \mathrm{TeV}$, Phys. Rev. Lett. 93 (2004) 162002 [hep-ex/0405004] [INSPIRE].

[4] LHCb collaboration, Observation of $J / \psi \phi$ structures consistent with exotic states from amplitude analysis of $B^{+} \rightarrow J / \psi \phi K^{+}$decays, Phys. Rev. Lett. 118 (2017) 022003 [arXiv: 1606.07895] [INSPIRE].

[5] LHCb collaboration, Amplitude analysis of $B^{+} \rightarrow J / \psi \phi K^{+}$decays, Phys. Rev. D 95 (2017) 012002 [arXiv: 1606.07898] [INSPIRE].

[6] CDF collaboration, Observation of the $Y(4140)$ Structure in the $J / \psi \phi$ Mass Spectrum in $B^{ \pm} \rightarrow J / \psi \phi K^{ \pm}$Decays, Mod. Phys. Lett. A 32 (2017) 1750139 [arXiv:1101.6058] [INSPIRE].

[7] LHCb collaboration, Observation of $J / \psi p$ Resonances Consistent with Pentaquark States in $\Lambda_{b}^{0} \rightarrow J / \psi K^{-} p$ Decays, Phys. Rev. Lett. 115 (2015) 072001 [arXiv:1507.03414] [INSPIRE].

[8] LHCb collaboration, Observation of a narrow pentaquark state, $P_{c}(4312)^{+}$and of two-peak structure of the $P_{c}(4450)^{+}$, Phys. Rev. Lett. 122 (2019) 222001 [arXiv:1904.03947] [INSPIRE].

[9] H.-X. Chen, W. Chen, X. Liu and S.-L. Zhu, The hidden-charm pentaquark and tetraquark states, Phys. Rept. 639 (2016) 1 [arXiv:1601.02092] [INSPIRE].

[10] A. Ali, J.S. Lange and S. Stone, Exotics: Heavy Pentaquarks and Tetraquarks, Prog. Part. Nucl. Phys. 97 (2017) 123 [arXiv:1706.00610] [InSPIRE].

[11] S.L. Olsen, T. Skwarnicki and D. Zieminska, Nonstandard heavy mesons and baryons: Experimental evidence, Rev. Mod. Phys. 90 (2018) 015003.

[12] F.K. Guo, C. Hanhart, U.G. Meißner, Q. Wang, Q. Zhao and B.S. Zou, Hadronic molecules, Rev. Mod. Phys. 90 (2018) 015004.

[13] M.B. Voloshin, Strange hadrocharmonium, Phys. Lett. B 798 (2019) 135022.

[14] K. Heikkila, S. Ono and N.A. Tornqvist, Heavy $c \bar{c}$ and $b \bar{b}$ quarkonium states and unitarity effects, Phys. Rev. D 29 (1984) 110 [Erratum ibid. D 29 (1984) 2136] [InSPIRE].

[15] M.R. Pennington and D.J. Wilson, Decay channels and charmonium mass-shifts, Phys. Rev. D 76 (2007) 077502 [arXiv:0704.3384] [INSPIRE].

[16] I.V. Danilkin and Y.A. Simonov, Dynamical origin and the pole structure of X(3872), Phys. Rev. Lett. 105 (2010) 102002 [arXiv:1006.0211] [INSPIRE].

[17] P.G. Ortega, J. Segovia, D.R. Entem and F. Fernandez, Coupled channel approach to the structure of the X(3872), Phys. Rev. D 81 (2010) 054023 [arXiv:0907.3997] [InSPIRE]. 
[18] P.G. Ortega, D.R. Entem and F. Fernandez, Molecular Structures in Charmonium Spectrum: The XYZ Puzzle, J. Phys. G 40 (2013) 065107 [arXiv:1205.1699] [inSPIRE].

[19] J. Ferretti, G. Galatà and E. Santopinto, Interpretation of the X(3872) as a charmonium state plus an extra component due to the coupling to the meson-meson continuum, Phys. Rev. C 88 (2013) 015207 [arXiv:1302.6857] [INSPIRE].

[20] J. Ferretti, G. Galatà and E. Santopinto, Quark structure of the $X(3872)$ and $\chi_{b}(3 P)$ resonances, Phys. Rev. D 90 (2014) 054010 [arXiv:1401.4431] [INSPIRE].

[21] J. Ferretti and E. Santopinto, Higher mass bottomonia, Phys. Rev. D 90 (2014) 094022 [arXiv:1306.2874] [INSPIRE].

[22] N.N. Achasov and E.V. Rogozina, $X(3872), I^{G}\left(J^{P C}\right)=0^{+}\left(1^{++}\right)$, as the $\chi_{1 c}(2 P)$ charmonium, Mod. Phys. Lett. A 30 (2015) 1550181 [arXiv:1501.03583] [INSPIRE].

[23] X.-W. Kang and J.A. Oller, Different pole structures in line shapes of the X(3872), Eur. Phys. J. C 77 (2017) 399 [arXiv: 1612.08420] [InSPIRE].

[24] Y. Lu, M.N. Anwar and B.-S. Zou, Coupled-Channel Effects for the Bottomonium with Realistic Wave Functions, Phys. Rev. D 94 (2016) 034021 [arXiv: 1606.06927] [InSPIRE].

[25] M.N. Anwar, Y. Lu and B.-S. Zou, $\chi_{b}(3 P)$ multiplet revisited: Hyperfine mass splitting and radiative transitions, Phys. Rev. D 99 (2019) 094005 [arXiv:1806.01155] [INSPIRE].

[26] J. Ferretti and E. Santopinto, Threshold corrections of $\chi_{c}(2 P)$ and $\chi_{b}(3 P)$ states and $J / \psi \rho$ and $J / \psi \omega$ transitions of the $\chi(3872)$ in a coupled-channel model, Phys. Lett. B 789 (2019) 550 [arXiv: 1806. 02489] [INSPIRE].

[27] J. Ferretti, E. Santopinto, M.N. Anwar and Y. Lu, Quark structure of the $\chi_{\mathrm{c}}(3 P)$ and $X(4274)$ resonances and their strong and radiative decays, arXiv:2002.09401 [INSPIRE].

[28] N.A. Tornqvist, From the deuteron to deusons, an analysis of deuteron-like meson meson bound states, Z. Phys. C 61 (1994) 525 [hep-ph/9310247] [INSPIRE].

[29] N.A. Tornqvist, Isospin breaking of the narrow charmonium state of Belle at 3872-MeV as a deuson, Phys. Lett. B 590 (2004) 209 [hep-ph/0402237] [InSPIRE].

[30] C. Hanhart, Y.S. Kalashnikova, A.E. Kudryavtsev and A.V. Nefediev, Reconciling the $X(3872)$ with the near-threshold enhancement in the $D^{0} \bar{D}^{* 0}$ final state, Phys. Rev. D 76 (2007) 034007 [arXiv:0704.0605] [INSPIRE].

[31] V. Baru, A.A. Filin, C. Hanhart, Y.S. Kalashnikova, A.E. Kudryavtsev and A.V. Nefediev, Three-body DD $\pi$ dynamics for the X(3872), Phys. Rev. D 84 (2011) 074029 [arXiv: 1108.5644] [INSPIRE].

[32] M.P. Valderrama, Power Counting and Perturbative One Pion Exchange in Heavy Meson Molecules, Phys. Rev. D 85 (2012) 114037 [arXiv:1204.2400] [INSPIRE].

[33] F. Aceti, R. Molina and E. Oset, The $X(3872) \rightarrow J / \psi \gamma$ decay in the $D \bar{D}^{*}$ molecular picture, Phys. Rev. D 86 (2012) 113007 [arXiv:1207.2832] [inSPIRE].

[34] F.-K. Guo, C. Hidalgo-Duque, J. Nieves and M.P. Valderrama, Consequences of Heavy Quark Symmetries for Hadronic Molecules, Phys. Rev. D 88 (2013) 054007 [arXiv:1303.6608] [INSPIRE].

[35] R.L. Jaffe, Multi-Quark Hadrons. 2. Methods, Phys. Rev. D 15 (1977) 281 [InSPIRE].

[36] B. Silvestre-Brac and C. Semay, Systematics of $L=0 q^{2} \bar{q}^{2}$ systems, Z. Phys. C 57 (1993) 273 [INSPIRE]. 
[37] D.M. Brink and F. Stancu, Tetraquarks with heavy flavors, Phys. Rev. D 57 (1998) 6778 [INSPIRE].

[38] L. Maiani, F. Piccinini, A.D. Polosa and V. Riquer, Diquark-antidiquarks with hidden or open charm and the nature of X(3872), Phys. Rev. D 71 (2005) 014028 [hep-ph/0412098] [INSPIRE].

[39] N. Barnea, J. Vijande and A. Valcarce, Four-quark spectroscopy within the hyperspherical formalism, Phys. Rev. D 73 (2006) 054004 [hep-ph/0604010] [INSPIRE].

[40] E. Santopinto and G. Galatà, Spectroscopy of tetraquark states, Phys. Rev. C 75 (2007) 045206 [hep-ph/0605333] [INSPIRE].

[41] D. Ebert, R.N. Faustov, V.O. Galkin and W. Lucha, Masses of tetraquarks with two heavy quarks in the relativistic quark model, Phys. Rev. D 76 (2007) 114015 [arXiv:0706.3853] [INSPIRE].

[42] D. Ebert, R.N. Faustov and V.O. Galkin, Relativistic description of heavy tetraquarks, Phys. Atom. Nucl. 72 (2009) 184 [arXiv:0802.1806] [inSPIRE].

[43] C. Deng, J. Ping and F. Wang, Interpreting $Z_{c}(3900)$ and $Z_{c}(4025) / Z_{c}(4020)$ as charged tetraquark states, Phys. Rev. D 90 (2014) 054009 [arXiv:1402.0777] [INSPIRE].

[44] L. Zhao, W.-Z. Deng and S.-L. Zhu, Hidden-Charm Tetraquarks and Charged $Z_{c}$ States, Phys. Rev. D 90 (2014) 094031 [arXiv: 1408.3924] [INSPIRE].

[45] Q.-F. Lü and Y.-B. Dong, X(4140), X(4274), X(4500) and X(4700) in the relativized quark model, Phys. Rev. D 94 (2016) 074007 [arXiv: 1607.05570] [InSPIRE].

[46] M.N. Anwar, J. Ferretti, F.-K. Guo, E. Santopinto and B.-S. Zou, Spectroscopy and decays of the fully-heavy tetraquarks, Eur. Phys. J. C 78 (2018) 647 [arXiv:1710.02540] [InSPIRE].

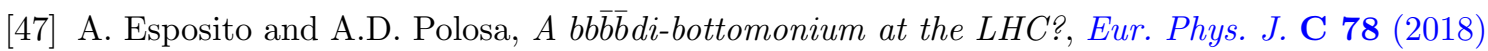
782 [arXiv: 1807.06040] [INSPIRE].

[48] M.N. Anwar, J. Ferretti and E. Santopinto, Spectroscopy of the hidden-charm $[q c][\bar{q} \bar{c}]$ and $[s c][\bar{s} \bar{c}]$ tetraquarks in the relativized diquark model, Phys. Rev. D 98 (2018) 094015 [arXiv: 1805.06276] [INSPIRE].

[49] G. Yang, J. Ping and J. Segovia, Doubly-heavy tetraquarks, Phys. Rev. D 101 (2020) 014001 [arXiv: 1911.00215] [INSPIRE].

[50] M.A. Bedolla, J. Ferretti, C.D. Roberts and E. Santopinto, Spectrum of fully-heavy tetraquarks from a diquark+antidiquark perspective, arXiv:1911.00960 [INSPIRE].

[51] F.-K. Guo, C. Hanhart and U.-G. Meissner, Evidence that the $Y(4660)$ is a f(0)(980)psi-prime bound state, Phys. Lett. B 665 (2008) 26 [arXiv:0803.1392] [INSPIRE].

[52] F.-K. Guo, C. Hanhart and U.-G. Meissner, Implications of heavy quark spin symmetry on heavy meson hadronic molecules, Phys. Rev. Lett. 102 (2009) 242004 [arXiv:0904.3338] [INSPIRE].

[53] S. Dubynskiy and M.B. Voloshin, Hadro-Charmonium, Phys. Lett. B 666 (2008) 344 [arXiv: 0803.2224] [INSPIRE].

[54] M.B. Voloshin, $Z_{c}(3900)$ - what is inside?, Phys. Rev. D 87 (2013) 091501 [arXiv: 1304.0380] [INSPIRE].

[55] X. Li and M.B. Voloshin, $Y(4260)$ and $Y(4360)$ as mixed hadrocharmonium, Mod. Phys. Lett. A 29 (2014) 1450060 [arXiv:1309.1681] [InSPIRE]. 
[56] Q. Wang et al., Y(4260): hadronic molecule versus hadro-charmonium interpretation, Phys. Rev. D 89 (2014) 034001 [arXiv: 1309.4303] [INSPIRE].

[57] M. Cleven, F.-K. Guo, C. Hanhart, Q. Wang and Q. Zhao, Employing spin symmetry to disentangle different models for the XYZ states, Phys. Rev. D 92 (2015) 014005 [arXiv: 1505.01771] [INSPIRE].

[58] N. Brambilla, G. Krein, J. Tarrús Castellà and A. Vairo, Long-range properties of $1 S$ bottomonium states, Phys. Rev. D 93 (2016) 054002 [arXiv:1510.05895] [INSPIRE].

[59] M.I. Eides, V.Y. Petrov and M.V. Polyakov, Narrow Nucleon- $\psi(2 S)$ Bound State and LHCb Pentaquarks, Phys. Rev. D 93 (2016) 054039 [arXiv:1512.00426] [INSPIRE].

[60] M.I. Eides, V.Y. Petrov and M.V. Polyakov, Pentaquarks with hidden charm as hadroquarkonia, Eur. Phys. J. C 78 (2018) 36 [arXiv:1709.09523] [INSPIRE].

[61] M.I. Eides, V.Y. Petrov and M.V. Polyakov, New LHCb pentaquarks as hadrocharmonium states, arXiv:1904.11616 [inSPIRE].

[62] I.A. Perevalova, M.V. Polyakov and P. Schweitzer, On LHCb pentaquarks as a baryon- $\psi(2 S)$ bound state: prediction of isospin- $\frac{3}{2}$ pentaquarks with hidden charm, Phys. Rev. D 94 (2016) 054024 [arXiv: 1607.07008] [INSPIRE].

[63] M. Alberti, G.S. Bali, S. Collins, F. Knechtli, G. Moir and W. Söldner, Hadroquarkonium from lattice QCD, Phys. Rev. D 95 (2017) 074501 [arXiv:1608.06537] [inSPIRE].

[64] J. Ferretti, $\eta_{\mathrm{c}^{-}}$and $J / \psi$-isoscalar meson bound states in the hadro-charmonium picture, Phys. Lett. B 782 (2018) 702 [arXiv:1805.04717] [INSPIRE].

[65] J. Ferretti, E. Santopinto, M. Naeem Anwar and M.A. Bedolla, The baryo-quarkonium picture for hidden-charm and bottom pentaquarks and $L H C b P_{\mathrm{c}}(4380)$ and $P_{\mathrm{c}}(4450)$ states, Phys. Lett. B 789 (2019) 562 [arXiv: 1807.01207] [INSPIRE].

[66] J.Y. Panteleeva, I.A. Perevalova, M.V. Polyakov and P. Schweitzer, Tetraquarks with hidden charm and strangeness as $\phi-\psi(2 S)$ hadrocharmonium, Phys. Rev. C 99 (2019) 045206 [arXiv: 1802.09029] [INSPIRE].

[67] M.B. Voloshin, Charmonium, Prog. Part. Nucl. Phys. 61 (2008) 455 [arXiv:0711.4556] [INSPIRE].

[68] S.J. Brodsky, I.A. Schmidt and G.F. de Teramond, Nuclear bound quarkonium, Phys. Rev. Lett. 64 (1990) 1011 [INSPIRE].

[69] A.B. Kaidalov and P.E. Volkovitsky, Heavy quarkonia interactions with nucleons and nuclei, Phys. Rev. Lett. 69 (1992) 3155 [INSPIRE].

[70] A. Sibirtsev and M.B. Voloshin, The Interaction of slow J/psi and psi' with nucleons, Phys. Rev. D 71 (2005) 076005 [hep-ph/0502068] [INSPIRE].

[71] K. Gottfried, Hadronic transitions between quark-antiquark bound states, Phys. Rev. Lett. 40 (1978) 598 [INSPIRE].

[72] M.B. Voloshin, On Dynamics of Heavy Quarks in Nonperturbative QCD Vacuum, Nucl. Phys. B 154 (1979) 365 [inSPIRE].

[73] T.-M. Yan, Hadronic Transitions Between Heavy Quark States in Quantum Chromodynamics, Phys. Rev. D 22 (1980) 1652 [InSPIRE].

[74] Particle Data Group collaboration, Review of Particle Physics, Phys. Rev. D 98 (2018) 030001 [INSPIRE]. 
[75] Y.-H. Chen and F.-K. Guo, Chromopolarizabilities of bottomonia from the $\Upsilon(2 S, 3 S, 4 S) \rightarrow \Upsilon(1 S, 2 S) \pi \pi$ transitions, Phys. Rev. D 100 (2019) 054035 [arXiv: 1906.05766] [INSPIRE].

[76] S. Godfrey and N. Isgur, Mesons in a Relativized Quark Model with Chromodynamics, Phys. Rev. D 32 (1985) 189 [INSPIRE].

[77] B. Kubis, T.R. Hemmert and U.-G. Meissner, Baryon form-factors, Phys. Lett. B 456 (1999) 240 [hep-ph/9903285] [INSPIRE].

[78] A.J. Buchmann and R.F. Lebed, Baryon charge radii and quadrupole moments in the $1 / N_{c}$ expansion: The three flavor case, Phys. Rev. D 67 (2003) 016002 [hep-ph/0207358] [INSPIRE].

[79] T. Ledwig, H.-C. Kim, A.J. Silva and K. Goeke, Electric properties of the baryon anti-decuplet in the SU(3) chiral quark-soliton model, Phys. Rev. D 74 (2006) 054005 [hep-ph/0603122] [INSPIRE].

[80] M.E. Carrillo-Serrano, W. Bentz, I.C. Cloët and A.W. Thomas, Baryon Octet Electromagnetic Form Factors in a confining NJLS model, Phys. Lett. B 759 (2016) 178 [arXiv: 1603.02741] [INSPIRE].

[81] R.P. Feynman, R.B. Leighton and M.L. Sands, The Feynman Lectures on Physics, Addison-Wesley Pub. Co. (1963)-(1965).

[82] J.M. Richard, The Nonrelativistic three-body problem for baryons, Phys. Rept. 212 (1992) 1 [INSPIRE].

[83] M. Anselmino, E. Predazzi, S. Ekelin, S. Fredriksson and D.B. Lichtenberg, Diquarks, Rev. Mod. Phys. 65 (1993) 1199 [INSPIRE].

[84] E. Santopinto, An Interacting quark-diquark model of baryons, Phys. Rev. C 72 (2005) 022201 [hep-ph/0412319] [INSPIRE].

[85] J. Ferretti, A. Vassallo and E. Santopinto, Relativistic quark-diquark model of baryons, Phys. Rev. C 83 (2011) 065204 [INSPIRE].

[86] E. Santopinto and J. Ferretti, Strange and nonstrange baryon spectra in the relativistic interacting quark-diquark model with a Gürsey and Radicati-inspired exchange interaction, Phys. Rev. C 92 (2015) 025202 [arXiv:1412.7571] [INSPIRE].

[87] W. Celmaster, H. Georgi and M. Machacek, Potential Model of Meson Masses, Phys. Rev. D 17 (1978) 879 [INSPIRE].

[88] S. Capstick and N. Isgur, Baryons in a Relativized Quark Model with Chromodynamics, Phys. Rev. D 34 (1986) 2809 [INSPIRE].

[89] J. Ferretti, Effective Degrees of Freedom in Baryon and Meson Spectroscopy, Few Body Syst. 60 (2019) 17 [INSPIRE]. 